\title{
Regioselective Dehydration of Sugar Thioacetals under Mild Conditions
}

\author{
Rachel Szpara, Alexander Goyder, Michael J. Porter, Helen C. Hailes, and Tom D. Sheppard*
}

Cite This: https://doi.org/10.1021/acs.orglett.1c00424

Read Online

\section{ACCESS | Lلlll Metrics \& More | 回 Article Recommendations | st Supporting Information}

\begin{abstract}
Sugars are abundant in waste biomass, making them sustainable chiral building blocks for organic synthesis. The demand for chiral saturated heterocyclic rings for pharmaceutical applications is increasing as they provide well-defined three-dimensional frameworks that show increased metabolic resistance. A range of sugar thioacetals can be dehydrated selectively at C-2 under mild basic conditions, and the resulting ketene thioacetals can be applied to the production of useful chiral building blocks via further selective dehydration reactions.
\end{abstract}

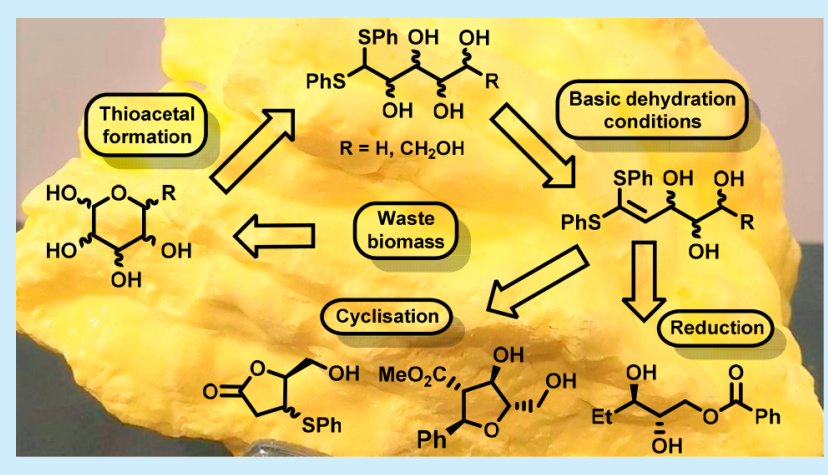

C arbohydrate biomass is an abundant renewable resource which has enormous potential for the synthesis of valuable chemical building blocks. ${ }^{1}$ The sugars present in this material are of particular interest as a functionalized carbon source to produce chiral saturated heterocycles which are of widespread potential utility in pharmaceutical development. ${ }^{2}$ While there are many well-established methods for converting sugars into chiral heterocycles such as tetrahydrofurans (THFs) and tetrahydropyrans (THPs), these typically rely on lengthy synthetic sequences involving the extensive use of protecting groups and high cost/energy reagents (e.g., $\mathrm{Tf}_{2} \mathrm{O}$ ). ${ }^{3}$ They are therefore somewhat resourceintensive and relatively inefficient approaches, especially for the large-scale preparation of chiral building blocks, and chiral heterocycles derived from sugars remain relatively underexplored in drug discovery applications. ${ }^{4}$ The development of more efficient and sustainable synthetic routes to chiral building blocks from sugars is therefore of great interest, particularly if the use of protecting groups and high-cost reagents can be minimized or avoided. In this context, the identification of reactions that can be used to achieve the regioselective dehydration of sugars without the need for protecting groups is particularly important. Notably, the selective removal of one or more hydroxyl groups from the sugar backbone will lead to molecules with inherently more useful properties for pharmaceutical applications.

There have been recent reports of selective transformations of unprotected sugars and their derivatives using both biocatalytic $^{5-8}$ and chemical approaches. ${ }^{9-11}$ Deoxygenation/ dehydration of sugars is of particular interest, and only a few approaches have been described. For example, Gagné has reported methods for the regioselective reductive cyclization of protected sugar-derived polyols 1 using silane reagents ${ }^{12,13}$ in the presence of Lewis acids such as $\mathrm{B}\left(\mathrm{C}_{6} \mathrm{~F}_{5}\right)_{3}$, leading to the formation of a range of chiral THFs and THPs 2 which can be accessed from sugars in a few steps (Scheme 1a).

In previous work, we have developed methods for the regioselective dehydration of sugar hydrazones, e.g., 3 (Scheme $1 \mathrm{~b})$, to give access to a range of chiral THFs (e.g., syn-4 and anti-4) under very mild conditions. ${ }^{14,15}$ These reactions are readily scalable and provided access to useful chiral building blocks in only a few steps. Importantly, it was also observed that cyclization of the sugar hydrazones under acidic or basic conditions provides complementary stereoselectivities. ${ }^{14}$ The acid-catalyzed cyclization takes place under thermodynamic control, most likely proceeding via the stabilized diazenium cation, whereas the base-mediated cyclization appears to involve a kinetically controlled $\mathrm{S}_{\mathrm{N}} 2$ ring-opening of a cyclic carbonate intermediate which can epimerize prior to cyclization. In this latter reaction, it was rationalized that the main role of the hydrazone is to hold the sugar in the openchain conformation which facilitates cyclization to the THF. We therefore envisaged that this approach could be extended to other open chain sugars such as thioacetals. Given that the formation of dimethylhydrazones from hexoses is often slow and relatively low-yielding, thioacetals might prove to be a more versatile alternative as they can readily be accessed from

Received: February 5, 2021 
Scheme 1. (a) Reductive cyclization of Silyl-Protected Sugars; ${ }^{11-13}$ (b) Chiral THF Formation via the Dehydration of Pentose Sugars; ${ }^{14,15}$ (c) This Work: Regioselective Dehydration of Sugar Thioacetals

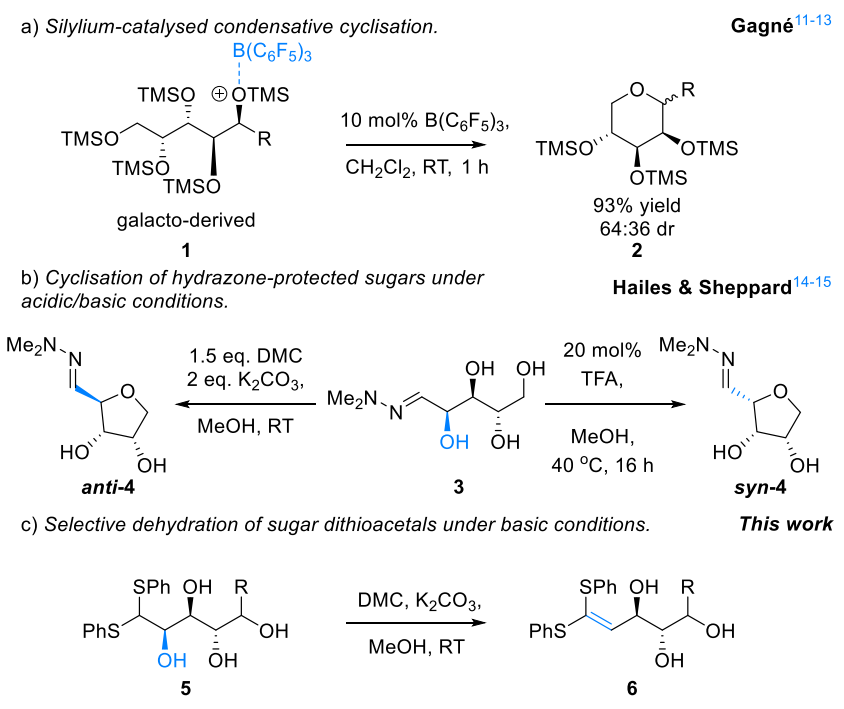

both pentoses and hexoses. In this paper, we describe methods for the regioselective dehydration of sugar thioacetals at C-2 and C-3 under mild and scalable conditions to provide access to novel chiral polyols and heterocycles (Scheme 1c).

Using L-arabinose, which is available from waste sugar beet pulp, ${ }^{15,96}$ as a test substrate, the corresponding ethyl and phenyl thioacetals were prepared via the reported procedures. ${ }^{17,18}$ Treatment of the ethyl thioacetal with $\mathrm{K}_{2} \mathrm{CO}_{3} /$ dimethyl carbonate (DMC) led to the formation of a complex mixture of products. However, reaction of the readily formed phenyl thioacetal $\mathbf{5 a}{ }^{18}$ under similar conditions led to the formation of the ketene thioacetal $\mathbf{6 a}$ as a single product. In addition, purification of the phenyl thioacetal derivative could be achieved via recrystallization, avoiding the need for column chromatography. Interestingly, unlike the reactions of the corresponding hydrazones, the THF was not formed, and a selective dehydration took place exclusively at the C-2 position to give alkene $6 \mathrm{a}$ in near-quantitative yield on a $5 \mathrm{~g}$ scale (Scheme 2).

Scheme 2. Thioacetal Protection of L-Arabinose Followed by Selective Dehydration under Mild Conditions ${ }^{14,18}$

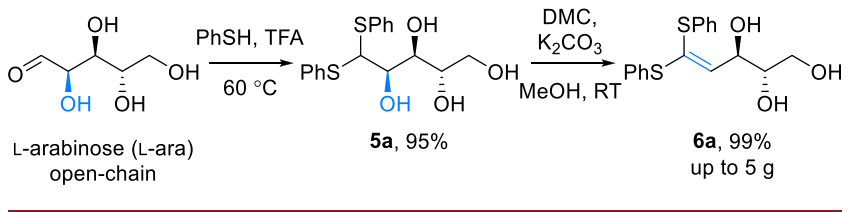

The $\mathrm{PhS}$ groups in 5a make the $\mathrm{C}$-1 proton fairly acidic, and hence, it is clear that an elimination reaction can take place readily when the C-2 hydroxyl group is activated by DMC. ${ }^{14}$ The formation of similar ketene dithioacetals has previously been reported as a problematic side reaction in reactions of protected derivatives with strong bases (e.g., sodium methylsulfinylmethylide or $n$-BuLi). ${ }^{19,20}$ Given that our reaction conditions are very mild, and that the reaction is selective and high yielding, this potentially offers a readily scalable method for the selective C-2 deoxygenation of sugars without the need for hydroxyl protecting groups. The scope of this approach was then explored (Scheme 3). Selective dehydration was carried out with an array of sugar dithioacetals derived from aldose sugars, in moderate to excellent yields (48-99\%) for several pentose and hexose sugars (6a, 6b, 6e, 6f). However, some thioacetals, such as those derived from $\mathrm{D}-$ ribose $(\mathbf{5 c})$, L-rhamnose $(\mathbf{5 d})$ and D-mannose $(\mathbf{5 g})$, gave little to no conversion to the alkene. A common feature of the unsuccessful substrates is anti-stereochemistry at the C-2 and C-3 positions. This potentially provides a useful insight into the mechanism of the reaction, which is likely to occur via (reversible) formation of a cyclic carbonate at C-2/C-3, through reaction of the polyol with dimethyl carbonate. This then subsequently undergoes elimination by removal of the acidic C-1 proton (Scheme 4).

The stereochemical relationship between the C-2 and C-3 alcohols may well affect the ease with which the carbonate can be formed (Scheme 4). As shown in structure 7c, sugars with anti stereochemistry at C-2/C-3 (e.g., D-rib) will have to form the more sterically hindered syn-cyclic carbonate. This hindered carbonate may also disfavor alignment of the C-1 proton into the correct orientation for the subsequent E-2 elimination. In contrast, sugars with syn-stereochemistry at C2/C-3 (L-ara) will form the less hindered anti-cyclic carbonate (e.g., 7a) which can easily adopt the required conformation for E-2 elimination to generate the alkene. DFT calculations at the M06-2X/6-31G(d,p) level confirmed that the free energy change in going from $5 \mathrm{a}$ to $7 \mathrm{a}$ in methanol solution is ca. $21 \mathrm{~kJ}$ $\mathrm{mol}^{-1}$ more negative than that going from $5 \mathrm{c}$ to $7 \mathrm{c}$.

Attempts to use more reactive electrophiles such as carbonyldiimidazole with $\mathbf{5 c}$ failed to give any improvement in the yield, indicating that the stereochemical relationship in these starting materials presents a significant barrier to successful dehydration under mild reaction conditions. An alternative strategy was therefore considered for anti-sugars which did not rely on the formation of a cyclic intermediate. It was envisaged that conversion of the thioacetal $\mathbf{5 c}$ to the corresponding peracetate could lead to sufficient activation of the $\mathrm{C}-2$ alcohol for it to act as a leaving group, facilitating dehydration under basic conditions. Formation of the peracetate derivatives with pyridine $/ \mathrm{Ac}_{2} \mathrm{O},{ }^{21}$ prior to treatment with a base was explored for the D-ribose, L-rhamnose, and Dmannose thioacetal derivatives (Scheme 5). Following acetylation, the protected sugars were stirred under basic conditions and monitored for ketene thioacetal formation. Although unreactive with $\mathrm{K}_{2} \mathrm{CO}_{3}$, the use of the stronger bases DBU (1,8-diazabicyclo[5.4.0] undec-7-ene), TBD (1,5,7triazabicyclo[4.4.0] dec-5-ene), and ${ }^{\mathrm{t}} \mathrm{BuOK}$ led to formation of the desired products $\mathbf{8 c}, \mathbf{8 d}$, and $\mathbf{8 g}$ in $47-96 \%$ yields. Different bases proved to be preferable for each example studied.

With a series of sugar-derived ketene dithioacetals in hand, we then went on to explore the reactivity of these novel compounds (Scheme 6). We envisaged that reductive desulfurization of the ketene acetal group could lead to valuable chiral polyols containing a stereogenic center bearing an ethyl group. Thus, reduction of the L-arabinose derivative 6a with Raney-Ni gave a triol 9, which was isolated as the corresponding benzoate ester derivative 10 in $94 \%$ overall yield (Scheme 6). Depending on the sugar used, chiral polyols of this general structure could be useful in the synthesis of natural products such as eicosatetraenoic acid (precursor 11), ${ }^{22}$ polysaccharides found in Gram-negative bacteria $12,{ }^{23}$ and 
Scheme 3. Selective Dehydration of Thioacetal-Protected Aldose Sugars at the C-2 Position under Basic Conditions ${ }^{a}$

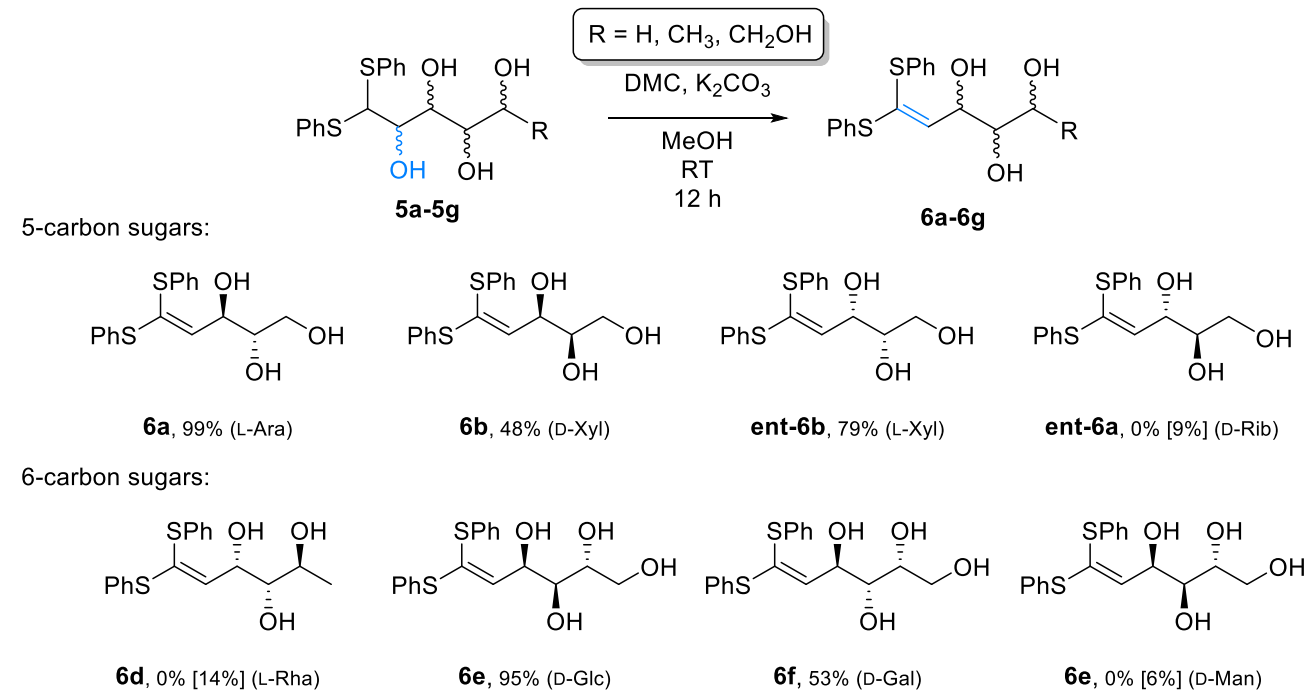

${ }^{a}$ Isolated yields. Conversions shown in brackets were determined by ${ }^{1} \mathrm{H}$ NMR spectroscopy using an internal standard of 1,4-dimethoxybenzene.

Scheme 4. Proposed Carbonate Intermediates in the Dehydration Sugar Thioacetals under Basic Conditions ${ }^{a}$

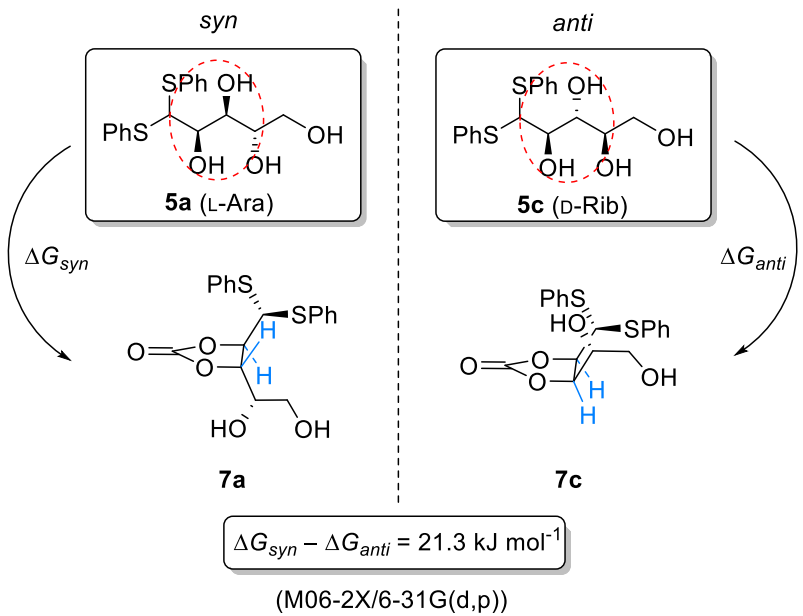

${ }^{a}$ DFT calculations suggest that the formation of a cyclic carbonate from a syn thioacetal $\mathbf{5 a}$ is considerably less endergonic than from an anti sugar thioacetal $\mathbf{5 c}$.

cholesterol side-chains (dihydroxyvitamins). ${ }^{24}$ In principle, the alkene in 6a has the potential to react with nucleophiles or electrophiles due to the ability of the two sulfur atoms to stabilize either an anion or a cation at C-1. However, it was not possible to observe any reactivity toward nucleophiles such as isopropylamine, morpholine, or sodium azide. Treatment of $\mathbf{6 a}$ with an "activated" aldehyde equivalent (benzaldehyde dimethyl acetal) under Lewis acidic conditions at high dilution (0.03 M) (Scheme 6) was then explored in the hope that condensation of one of the hydroxyl groups would deliver the electrophile to the dithioalkene leading to an intramolecular ring-closure reaction. Pleasingly, this yielded the cyclized methyl ester 13 as a single diastereoisomer but in low yield (unoptimized). Ester 13 is presumably formed by trapping of the dithiolium cation with methanol followed by hydrolytic cleavage of the $\mathrm{C}-\mathrm{S}$ bonds.

We also hypothesized that the allylic alcohol in ketene acetals 6 might be activated by the adjacent electron-rich
Scheme 5. Selective Dehydration of the Anti Sugar Thioacetals via Initial Acetylation ${ }^{21}$ Followed by BaseMediated Elimination ${ }^{a}$

$$
\text { ic }
$$

${ }^{a}$ Isolated yields are given for acetylation and dehydration steps, respectively. Conversion shown in brackets was determined by ${ }^{1} \mathrm{H}$ NMR spectroscopy using an internal standard of 1,4-dimethoxybenzene.

alkene, making further selective dehydration at C-3 possible. Treatment of arabinose-derived thioalkene $\mathbf{6 a}$ with $\operatorname{In}(\mathrm{OTf})_{3}$ led to cyclization at $\mathrm{C}$-1, presumably via a stabilized allylic cation. This leads via hydrolysis to the $\alpha, \beta$-unsaturated lactone which subsequently reacts with the liberated thiophenol to yield a diastereomeric mixture of known lactones 14 in 53\% yield (unoptimized). Lactones $\mathbf{1 4}$ have been widely employed previously as building blocks for asymmetric synthesis ${ }^{25,26}$ directed toward natural products e.g. intermediate in Branimycin synthesis $\mathbf{1 5}$ (Scheme 6). ${ }^{25}$ Previously reported syntheses of lactones 14 are lengthy (6 steps) and required the use of harsh workup procedures and toxic solvents. ${ }^{27}$ In contrast, using our procedure, we were able to produce 14 in only three steps with recrystallization being the main method of purification.

In summary, we have developed scalable methods for the regioselective C-2 dehydration of sugar thioacetals. ${ }^{28}$ The resulting ketene thioacetals are versatile synthetic intermediates $^{29}$ which can be used to access polyols containing a stereogenic center bearing an ethyl group. Preliminary studies 
Scheme 6. Further Reactions of L-Arabinose Ketene Thioacetal 6a to Access Chiral Building Blocks ${ }^{a}$

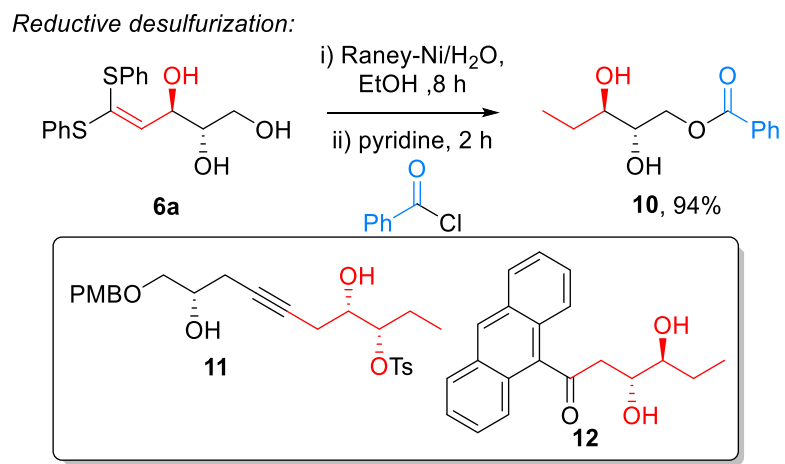

Nucleophilic reactivity:

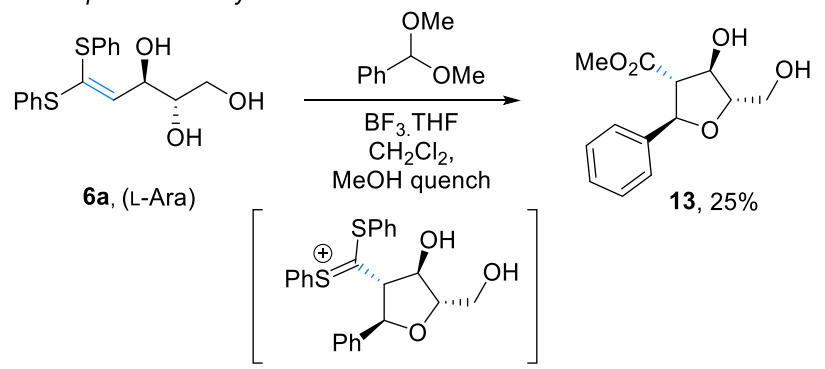

Lewis acid-mediated dehydration:

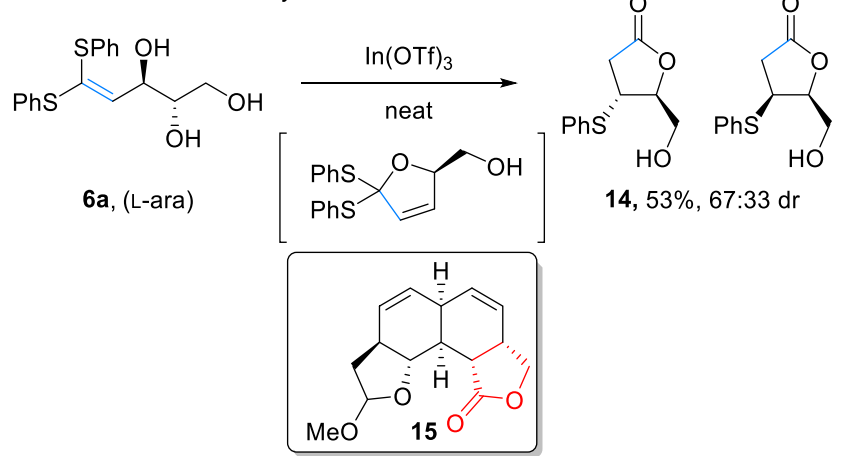

${ }^{a}$ Chiral motifs found in useful organic molecules are highlighted.

have also demonstrated that further selective dehydration reactions and cyclization of these compounds can be used to access chiral heterocycles (THFs, butyrolactones) that are useful building blocks for asymmetric synthesis.

\section{ASSOCIATED CONTENT}

\section{Supporting Information}

The Supporting Information is available free of charge on the ACS Publications Web site. Experimental procedures, details of DFT calculations including xyz coordinates for all calculated structures and ${ }^{1} \mathrm{H} /{ }^{13} \mathrm{C}$ NMR spectra for novel compounds. The Supporting Information is available free of charge at https://pubs.acs.org/doi/10.1021/acs.orglett.1c00424.

Experimental procedures, details of DFT calculations and ${ }^{1} \mathrm{H} /{ }^{13} \mathrm{C}$ NMR spectra for novel compounds (PDF) $x y z$ coordinates for all calculated structures (ZIP)

\section{AUTHOR INFORMATION}

\section{Corresponding Author}

Tom D. Sheppard - Department of Chemistry, Christopher Ingold Laboratories, University College London, London
WC1H 0AJ, U.K.; 다이.org/0000-0002-3455-1164; Email: tom.sheppard@ucl.ac.uk

Authors

Rachel Szpara - Department of Chemistry, Christopher Ingold Laboratories, University College London, London WC1H OAJ, U.K.; (1) orcid.org/0000-0003-1606-1660

Alexander Goyder - Department of Chemistry, Christopher Ingold Laboratories, University College London, London WC1H 0AJ, U.K.

Michael J. Porter - Department of Chemistry, Christopher Ingold Laboratories, University College London, London WC1H 0AJ, U.K.; (1) orcid.org/0000-0002-0376-5434

Helen C. Hailes - Department of Chemistry, Christopher Ingold Laboratories, University College London, London WC1H OAJ, U.K.; 10 orcid.org/0000-0001-5574-4742

Complete contact information is available at: https://pubs.acs.org/10.1021/acs.orglett.1c00424

\section{Notes}

The authors declare no competing financial interest.

\section{ACKNOWLEDGMENTS}

This work was supported by an EPSRC studentship provided by UCL Department of Chemistry (EP/R513143/1) and EPSRC funding for a $700 \mathrm{MHz} \mathrm{NMR}$ spectrometer (EP/ P020410/1).

\section{REFERENCES}

(1) Ruppert, A. M.; Weinberg, K.; Palkovits, R. Hydrogenolysis Goes Bio: From Carbohydrates and Sugar Alcohols to Platform Chemicals. Angew. Chem., Int. Ed. 2012, 51, 2564-2601.

(2) Ritter, T. K.; Wong, C.-H. Carbohydrate-Based Antibiotics: A New Approach to Tackling the Problem of Resistance. Angew. Chem., Int. Ed. 2001, 40, 3508-3533.

(3) Pétursson, S. Protecting Groups in Carbohydrate Chemistry. J. Chem. Educ. 1997, 74, 1297-1303.

(4) Garidel, P.; Brandenburg, K. Current Understanding of Polymyxin B Applications in Bacteraemia/Sepsis Therapy Prevention: Clinical, Pharmaceutical, Structural and Mechanistic Aspects. AntiInfect. Agents Med. Chem. 2009, 8, 367-385.

(5) Chang, S. W.; Shaw, J. F. Biocatalysis for the Production of Carbohydrate Esters. New Biotechnol. 2009, 26, 109-116.

(6) Fischer, F.; Happe, M.; Emery, J.; Fornage, A.; Schütz, R. Enzymatic Synthesis of 6- and 6'-O-Linoleyl- $\alpha$-d-Maltose: From Solvent-Free to Binary Ionic Liquid Reaction Media. J. Mol. Catal. B: Enzym. 2013, 90, 98-106.

(7) Abdulmalek, E.; Hamidon, N. F.; Abdul Rahman, M. B. Optimization and Characterization of Lipase Catalysed Synthesis of Xylose Caproate Ester in Organic Solvents. J. Mol. Catal. B: Enzym. 2016, 132, 1-4.

(8) Subrizi, F.; Benhamou, L.; Ward, J. M.; Sheppard, T. D.; Hailes, H. C. Aminopolyols from Carbohydrates: Amination of Sugars and Sugar-Derived Tetrahydrofurans with Transaminases. Angew. Chem., Int. Ed. 2019, 58, 3854-3858.

(9) Johnson, S.; Bagdi, A. K.; Tanaka, F. C-Glycosidation of Unprotected $\mathrm{Di}$ - and Trisaccharide Aldopyranoses with Ketones Using Pyrrolidine-Boric Acid Catalysis. J. Org. Chem. 2018, 83, 45814597.

(10) Bender, T. A.; Dabrowski, J. A.; Gagné, M. R. Homogeneous Catalysis for the Production of Low-Volume, High-Value Chemicals from Biomass. Nat. Rev. Chem. 2018, 2, 35-46.

(11) Adduci, L. L.; Bender, T. A.; Dabrowski, J. A.; Gagné, M. R. Chemoselective Conversion of Biologically Sourced Polyols into Chiral Synthons. Nat. Chem. 2015, 7, 576-581. 
(12) Bender, T. A.; Dabrowski, J. A.; Zhong, H.; Gagné, M. R. Diastereoselective $\mathrm{B}\left(\mathrm{C}_{6} \mathrm{~F}_{5}\right)_{3}$-Catalyzed Reductive Carbocyclization of Unsaturated Carbohydrates. Org. Lett. 2016, 18, 4120-4123.

(13) Lowe, J. M.; Seo, Y.; Gagné, M. R. Boron-Catalyzed SiteSelective Reduction of Carbohydrate Derivatives with Catecholborane. ACS Catal. 2018, 8, 8192-8198.

(14) Foster, R. W.; Tame, C. J.; Bučar, D. K.; Hailes, H. C.; Sheppard, T. D. Sustainable Synthesis of Chiral Tetrahydrofurans through the Selective Dehydration of Pentoses. Chem. - Eur. J. 2015, 21, 15947-15950.

(15) Benhamou, L.; Foster, R. W.; Ward, D. P.; Wheelhouse, K.; Sloan, L.; Tame, C. J.; Bučar, D. K.; Lye, G. J.; Hailes, H. C.; Sheppard, T. D. Functionalised Tetrahydrofuran Fragments from Carbohydrates or Sugar Beet Pulp Biomass. Green Chem. 2019, 21, 2035-2042.

(16) Cárdenas-Fernández, M.; Hamley-Bennett, C.; Leak, D. J.; Lye, G. J. Continuous Enzymatic Hydrolysis of Sugar Beet Pectin and LArabinose Recovery within an Integrated Biorefinery. Bioresour. Technol. 2018, 269, 195-202.

(17) Zinner, H. Notiz Uber Mercaptale Der D-Ribose. Chem. Ber. 1950, 83, 275-277.

(18) Funabashi, M.; Arai, S.; Shinohara, M. Novel Syntheses of Diphenyl and/or Trimethylene Dithioacetals of Mono- and Oligosaccharides in 90\% Trifluoroacetic Acid. J. Carbohydr. Chem. 1999, 18, 333-341.

(19) Horton, D.; Wander, J. D. Conformation of Acyclic Derivatives of Sugars. XI. Conformations of the D-Aldopentose Diethyl and Diphenyl Dithioacetals in Solution. J. Org. Chem. 1974, 39, 18591863.

(20) Ermanis, K.; Hsiao, Y. T.; Kaya, U.; Jeuken, A.; Clarke, P. A. The Stereodivergent Formation of 2,6-Cis and 2,6-Trans-Tetrahydropyrans: Experimental and Computational Investigation of the Mechanism of a Thioester Oxy-Michael Cyclization. Chem. Sci. 2017, $8,482-490$.

(21) Mukherjee, D.; Shah, B. A.; Gupta, P.; Taneja, S. C. Tandem Acetalation-Acetylation of Sugars and Related Derivatives with Enolacetates under Solvent-Free Conditions. J. Org. Chem. 2007, $72,8965-8968$.

(22) Goto, T.; Urabe, D.; Isobe, Y.; Arita, M.; Inoue, M. Total Synthesis of Four Stereoisomers of $(5 Z, 8 Z, 10 E, 14 Z)-12$-Hydroxy17,18-Epoxy-5,8,10,14-Eicosatetraenoic Acid and Their Anti-Inflammatory Activities. Tetrahedron 2015, 71, 8320-8332.

(23) Elloumi, N.; Aguiar, L.; Capmau, M. L. Exiton Chirality Method: A Tool for the Chirality Determination of 7,8-dideoxy-Lglycero-D-manno-oct-7-ynopyranose. Bull. Soc. Chim. Belg. 1994, 103, 647-650.

(24) Koizumi, N.; Ishiguro, M.; Yasuda, M.; Ikekawa, N. Stereoselective Introduction of Hydroxy Groups into the Cholesterol Side Chain. Preparation of (24R)- and (24S)-24,25-Dihydroxy- and (25R)- and (25S)-25,26-Dihydroxyvitamin D3 by Asymmetric Synthesis. J. Chem. Soc., Perkin Trans. 1 1983, 1, 1401-1410.

(25) Felzmann, W.; Arion, V. B.; Mieusset, J. L.; Mulzer, J. A Tether Controlled Exo-Selective Trans-Annular Diels-Alder (TADA) Reaction. Org. Lett. 2006, 8, 3849-3851.

(26) Ellermann, M.; Paulini, R.; Jakob-Roetne, R.; Lerner, C.; Borroni, E.; Roth, D.; Ehler, A.; Schweizer, W. B.; Schlatter, D.; Rudolph, M. G.; Diederich, F. Molecular Recognition at the Active Site of Catechol-O-Methyltransferase (COMT): Adenine Replacements in Bisubstrate Inhibitors. Chem. - Eur. J. 2011, 17, 6369-6381.

(27) Takano, S.; Kurotaki, A.; Takahashi, M.; Ogasawara, K. Practical Synthesis of Some Versatile Chiral Building Blocks from (D)-Mannitol. Synthesis 1986, 403-406.

(28) Szpara, R.; Goyder, A.; Porter, M. J.; Hailes, H. C.; Sheppard, T. D. Regioselective Dehydration of Sugar Thioacetals under Mild Conditions. ChemRxiv 2020, DOI: 10.26434/chemrxiv.13014371.v1.

(29) Wong, M. Y. H.; Gray, G. R. 2-Deoxypentoses. Stereoselective Reduction of Ketene Dithioacetals. J. Am. Chem. Soc. 1978, 100, $3548-3553$ 